\title{
Evidence for classification of c.1852_1853AA>GC in MLH1 as a neutral variant for Lynch syndrome
}

\author{
Adela Castillejo ${ }^{1}$, Carla Guarinos ${ }^{1}$, Ana Martinez-Canto ${ }^{1}$, Victor-Manuel Barbera', Cecilia Egoavil', \\ Maria-Isabel Castillejo ${ }^{1}$, Lucia Perez-Carbonell ${ }^{2}$, Ana-Beatriz Sanchez-Heras ${ }^{3}$, Angel Segura ${ }^{4}$, Enrique Ochoa ${ }^{5}$, \\ Rafael Lazaro ${ }^{6}$, Clara Ruiz-Ponte ${ }^{7}$, Luis Bujanda ${ }^{8}$, Montserrat Andreu ${ }^{9}$, Antoni Castells ${ }^{10}$, Angel Carracedo ${ }^{7}$, \\ Xavier Llor ${ }^{11}$, Juan Clofent ${ }^{12}$, Cristina Alenda ${ }^{13}$, Artemio Paya ${ }^{13}$, Rodrigo Jover ${ }^{14}$, Jose-Luis Soto ${ }^{{ }^{*}}$
}

\begin{abstract}
Background: Lynch syndrome (LS) is an autosomal dominant inherited cancer syndrome characterized by early onset cancers of the colorectum, endometrium and other tumours. A significant proportion of DNA variants in LS patients are unclassified. Reports on the pathogenicity of the c.1852_1853AA>GC (p.Lys618Ala) variant of the MLH1 gene are conflicting. In this study, we provide new evidence indicating that this variant has no significant implications for LS.

Methods: The following approach was used to assess the clinical significance of the p.Lys618Ala variant: frequency in a control population, case-control comparison, co-occurrence of the p.Lys618Ala variant with a pathogenic mutation, co-segregation with the disease and microsatellite instability in tumours from carriers of the variant. We genotyped p.Lys618Ala in 1034 individuals (373 sporadic colorectal cancer [CRC] patients, 250 index subjects from families suspected of having LS [revised Bethesda guidelines] and 411 controls). Three well-characterized LS families that fulfilled the Amsterdam II Criteria and consisted of members with the p.Lys618Ala variant were included to assess co-occurrence and co-segregation. A subset of colorectal tumour DNA samples from 17 patients carrying the p.Lys618Ala variant was screened for microsatellite instability using five mononucleotide markers.
\end{abstract}

Results: Twenty-seven individuals were heterozygous for the p.Lys618Ala variant; nine had sporadic CRC (2.41\%), seven were suspected of having hereditary CRC (2.8\%) and 11 were controls (2.68\%). There were no significant associations in the case-control and case-case studies. The p.Lys618Ala variant was co-existent with pathogenic mutations in two unrelated LS families. In one family, the allele distribution of the pathogenic and unclassified variant was in trans, in the other family the pathogenic variant was detected in the MSH6 gene and only the deleterious variant co-segregated with the disease in both families. Only two positive cases of microsatellite instability $(2 / 17,11.8 \%)$ were detected in tumours from p.Lys618Ala carriers, indicating that this variant does not play a role in functional inactivation of MLH1 in CRC patients.

Conclusions: The p.Lys618Ala variant should be considered a neutral variant for LS. These findings have implications for the clinical management of CRC probands and their relatives.

\section{Background}

Genetic testing is conducted for diverse purposes, including confirmation of diagnosis, risk prediction, carrier testing and reproductive decision-making. The identification of germ-line mutations in patients with inherited cancer syndromes enables them to be included in cancer

\footnotetext{
* Correspondence: soto_jos@gva.es

'Laboratorio Investigación. Hospital Universitario Elche. Elche. Spain

Full list of author information is available at the end of the article
}

surveillance programmes. Such programmes are effective in reducing cancer mortality in the families concerned. Moreover, family members who do not carry the mutation can be treated safely as low-risk individuals, avoiding unnecessary screening and preventing anxiety in the individuals concerned. Unfortunately, the number of suspected familial cancer cases in which a causative mutation is identified is far from ideal. All members from a family with a strong history of cancer and no causative mutation

\section{Biomed Central}


detected are included in a surveillance program. Identification of mutations depends on the specific syndrome and the criteria applied to select patients for genetic analyses.

The results of sequence-based genetic tests may be reported to physicians as: 1) positive, in which a mutation that clearly disrupts gene function is detected and is highly likely to have clinical consequences; 2) a genetic variant is detected but it is not known whether the variant has any effect on gene function that might confer an increased cancer risk (these variants are known as variants of uncertain/unclassified significance or unclassified variants [UVs]); and 3) negative, in which deleterious variant or UV is detected [1].

The majority of UVs are missense mutations or small in-frame deletions. The human gene pool harbours a vast number of rare missense substitutions, $70 \%$ of which are at least mildly deleterious [2]. Integration of various lines of evidence may help to classify UVs. Information on: 1) frequencies in cases and controls, 2) cooccurrence (in trans) with deleterious mutations, 3) cosegregation with disease in pedigrees, 4) pathological factors, 5) amino acid polarity or size, 6) evolutionary conservation of the residue, 7) splice predictions and 8) in vitro and/or in vivo functional assays may enable UVs to be classified as pathogenic or non-pathogenic [3].

Lynch syndrome (MIM\# 120435) (LS) is an autosomal dominant inherited cancer syndrome characterized by early onset colorectal cancer (CRC), cancer of the endometrium and tumours of the stomach, pancreas, small intestine, ovary, bladder and bile duct [4]. LS-associated tumours are characterized by DNA mismatch repair (MMR) deficiency, which may be evidenced by microsatellite instability (MSI) or loss of expression of MMR proteins using immunohistochemistry [5]. The proportion of genetic UVs in LS varies from $1 / 5$ to $1 / 3$ of all unique variants detected [6].

The MLH1 p.Lys618Ala (c.1852_1853AA>GC) variant was initially considered a deleterious variant based on its recurrent presence in LS families, in silico predictions and in vitro experiments on its functional effect. However, recent data on its co-segregation with LS have cast doubt on its clinical significance [6]. In this study, we provide evidence supporting the contention that this variant has no significant implications in LS.

The following approach was used to assess the clinical significance of the p.Lys618Ala variant: frequency in a control population, case-control and case-case comparisons, co-occurrence with a pathogenic mutation, co-segregation with the disease and MSI in tumours from carrier individuals.

\section{Methods}

\section{Controls and sporadic and familial CRC cases}

We genotyped the p.Lys618Ala variant in $M L H 1$ in 1034 individuals (373 sporadic CRC patients, 250 index subjects from families suspected of having LS [revised Bethesda Guidelines] and 411 controls). The controls were selected from the same hospitals, had no personal histories of cancer and had diagnoses unrelated to the variables of interest. They were matched for age, gender and race/ethnicity with the sporadic CRC patients.

No familial history of cancer was available from the control group. Patients diagnosed at an age over 50 years and not referred to Genetic Counselling Units were considered as sporadic CRC. Samples from sporadic CRC patients were obtained from the Elche University Hospital BioBank and the Castellon Provincial Hospital BioBank. Written consent to be included in the respective biobanks was obtained from each patient. CRC patients, as index subjects from families with suspicion of LS that attended Genetic Counselling at the Cancer Units of the Elche and La Fe Hospitals, were recruited. The study was approved by the Ethics Committee of the Elche University Hospital.

The median age of patients in the sporadic CRC group was 70 years (range, 52-93 years), 47 years (range, 21-87 years) for the familial group and 71 years (range, 25-96 years) for the controls. The sex distribution was 58\% men and $42 \%$ women for the sporadic CRC group and $53.3 \%$ men and $46.7 \%$ women for the controls.

\section{Families carrying the p.Lys618Ala variant}

Three characterized LS families that fulfilled the Amsterdam II Criteria and that consisted of members with the p.Lys618Ala variant were included to assess cooccurrence and co-segregation. Two families attended the Genetic Counselling in Cancer Units of the Elche and La Fe Hospitals and one family was a member of the EPICOLON cohort [7].

Concomitant deleterious variants were detected in two of the families: one in the $M L H 1$ gene (c.676C $>\mathrm{T}$; p . Arg226X) and the other in the MSH6 gene (c.3013C >T; p.Arg1005X). Seventeen affected and unaffected family members from these two families were tested for the pathogenic and p.Lys618Ala variants.

\section{Genotyping of the MLH1 p.Lys618Ala variant}

DNA from blood cells (familial cancer cases and controls) or colorectal mucosa of normal appearance (sporadic cases) was used for the c.1852_1853AA>GC variant genotyping. This was assessed using the iPLEX Gold method (Sequenom, CA, USA), in which singlebase extension and MALDI-TOF technology are employed for allelic discrimination. These experiments were carried out at the Centro Español de Genotipado (CEGEN) genotyping platform facilities. Quality control for genotyping was conducted by direct sequencing of familial cancer subjects who underwent genetic analysis for MLH1 (49/1034, 4.7\%). 
Microsatellite instability and MLH1 immunohistochemical expression

A subset of colorectal tumour DNA samples from 17 patients carrying the p.Lys618Ala variant (eight from the familial group and nine from the sporadic CRC group) was screened for MSI status using five mononucleotide markers (BAT26, BAT25, NR21, NR24 and NR27) and multiplex PCR as previously described by Buhard et al [8].

Tumours from p.Lys618Ala carrier cases in the familial group (seven index subjects and one relative) were also analysed for MLH1 protein expression using immunohistochemistry and anti-MLH1 antibodies (PharMingen, CA, USA) as described elsewhere [7]. Tumour cells were judged negative for protein expression only if they lacked staining in a sample in which normal colonocytes and stroma cells were stained. If no immunostaining of normal tissue could be demonstrated, the results were considered unreliable.

MLH1 promoter hypermethylation by Methylation Sensitive Multiplex Ligation-dependent Probe Amplification (MS-MLPA), and BRAF p.Val600Glu mutation by direct sequencing from tumor DNA was also assess when MLH1 loss of expression was detected.

\section{Statistical analysis}

Hardy-Weinberg equilibrium was calculated for the control, sporadic CRC and familial CRC groups. Allelic and genotype frequencies were calculated. In the case-control study of sporadic CRC, we estimated the odds ratio (OR) and 95\% confidence interval (95\% CI) for the p. Lys618Ala variant using unconditional logistic regression adjusted for age and sex. We analysed for potential effect modification by age using an analysis stratified according to median age at diagnosis for the sporadic CRC cases ( $\leq 70$ years or $>70$ years). A $\chi^{2}$ test was used to evaluate differences in p.Lys618Ala carrier frequencies between the tumour and control groups. A probability level of $<0.05$ was considered significant.

\section{Results}

No discordances were detected in the genotyping quality control. The genotype distributions in the control, sporadic and familial CRC populations did not deviate significantly from that expected for a population in Hardy-Weinberg equilibrium (Table 1).

Twenty-seven individuals were heterozygous for the $\mathrm{p}$. Lys618Ala variant (Figure 1); 11 were controls (11/411, $2.68 \%)$, nine were CRC patients from the sporadic group $(9 / 373,2.41 \%)$ and seven were CRC patients from the familial group $(7 / 250,2.8 \%)$. None of the individuals was homozygous for the minor allele.

There were no significant associations in the case-control and case-case studies $(80 \%$ detection power, OR =
Table 1 Allelic and genotypic frequencies and HardyWeinberg equilibrium

\begin{tabular}{ccccc}
\hline & $\begin{array}{c}\text { Controls } \\
\mathbf{n}=\mathbf{4 1 1}\end{array}$ & $\begin{array}{c}\text { Sporadic CRC } \\
\mathbf{n}=\mathbf{3 7 3}\end{array}$ & $\begin{array}{c}\text { Familial CRC } \\
\mathbf{n}=\mathbf{2 5 0}\end{array}$ & $\begin{array}{c}\text { Total } \\
\mathbf{n}=\mathbf{1 0 3 4}\end{array}$ \\
\hline $\begin{array}{c}\text { Allele } \\
\text { frequencies }\end{array}$ & & & & \\
AA & 0.9866 & 0.9879 & 0.9860 & 0.9869 \\
GC & 0.0134 & 0.0121 & 0.0140 & 0.0131 \\
\hline $\begin{array}{c}\text { Genotype } \\
\text { frequencies }\end{array}$ & & & & \\
AA/AA & 0.9732 & 0.9759 & 0.9720 & 0.9739 \\
AA/GC & 0.0268 & 0.0241 & 0.0280 & 0.0261 \\
GC/GC & 0 & 0 & 0 & 0 \\
\hline $\begin{array}{c}\text { HW } \\
\text { equilibrium }\end{array}$ & 0.9639 & 0.9706 & 0.9698 & 0.9448 \\
(p) & & & & \\
\hline
\end{tabular}

*Individuals from apparently unrelated families.

3.0; two-sided test, alpha level $=5 \%$ ) (Table 2) and no statistically significant associations when the OR was adjusted for age and sex.

In one of the families with LS, the index subject was heterozygous for a pathogenic $M L H 1$ variant (c.767C > T; p.Arg226X) and the p.Lys618Ala variant. Two of his offspring, who were diagnosed with CRC at the ages of 36 and 39 years, carried the deleterious variant but not the p.Lys618Ala variant. An unaffected daughter (III-12) carried the p.Lys618Ala variant but not the deleterious variant. Two nephews (III-3; III-4) were also diagnosed with CRC at the ages of 30 and 42 years and they carried only the deleterious variant. Two other healthy nephews (III-6; III-7) had the wild types of the two variants (Family \#1, Figure 2).

In the second LS family, the index subject, one sister and one brother with CRC (II-5; II-6; II-7, respectively) had a deleterious variant in MSH6 (c.3013C > T; p. Arg1005X) but did not have the p.Lys618Ala variant. This variant was present in only three of four unaffected

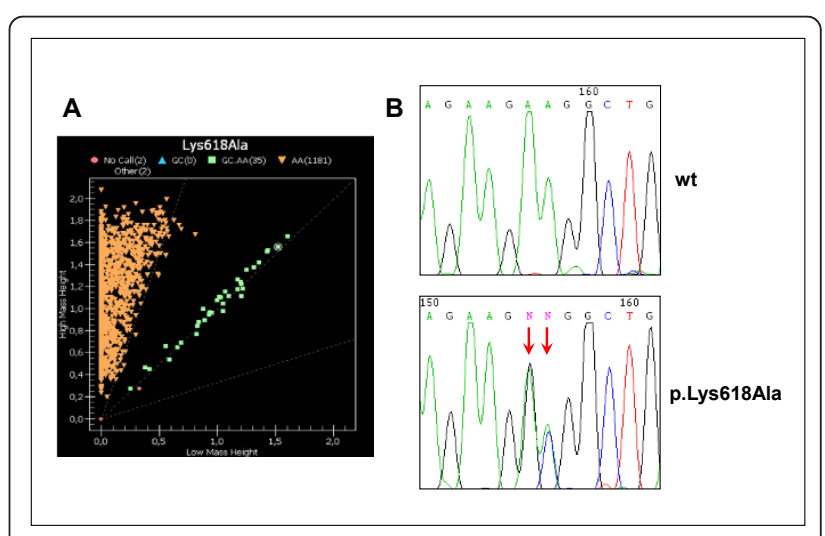

Figure 1 Results of genotyping for the p.Lys618Ala variant using the iPLEX Sequenom (A) and sequencing (B) methods. 
Table 2 Results of case-control and case-case analyses. Odds ratios (ORs) and $95 \%$ confidence intervals (95\% Cls) for the p.Lys618Ala variant

\begin{tabular}{cccc}
\hline & $\begin{array}{c}\text { Sporadic vs } \\
\text { Controls }\end{array}$ & $\begin{array}{c}\text { Familial vs } \\
\text { Controls }\end{array}$ & $\begin{array}{c}\text { Sporadic vs } \\
\text { Familial }\end{array}$ \\
\hline $\begin{array}{c}\text { OR } \\
{[95 \% \mathrm{Cl}]}\end{array}$ & $0.899[0.378-2.139]$ & $1.048[0.414-2.655]$ & $0.955[0.377-2.417]$ \\
\hline $\mathbf{p}$ value & 1 & 1 & 1 \\
\hline
\end{tabular}

nephews (III-2; III-3; III-4) and was inherited from the parental branch, in which there was no familial history of cancer. Individuals III-3 and III-4 inherited also the deleterious variant. No genetic testing was available from the father or paternal relatives (Family \#2, Figure 3).

The p.Lys618Ala variant was present in the third family that fulfilled the Amsterdam II Criteria. A firstgrade familiar non-carrier of this variant was diagnosed with a colonic polyp with a high grade of dysplasia at the age of 39 years and with four colonic polyps at the age of 42 years (Family \#3, Figure 4).

Of the 17 CRC patients with the Lys618Ala variant, two had MSI (11.8\%), one in the familial CRC group $(1 / 8)$ and one in the sporadic CRC group (1/9).

The MSI-positive patient from the familial CRC group showed loss of immunohistochemical expression of MLH1. This is the index subject (II-3) for the third family (Figure 4) and no hypermethylation of $M L H 1$ gene promoter; no BRAF p.Val600Glu mutation were detected in this case.

\section{Discussion}

The accelerated development of genetic counselling in cancer during the past few years is due to the feedback and interactive information sharing on genetic studies, clinical management and psychological issues in families with a high risk of cancer. Identification of deleterious variants in such families is essential for accurate assessment of individual risk and, if required, subsequent inclusion into a personalized surveillance programme.

Unfortunately, genetic testing for hereditary cancer frequently fails to identify unambiguous deleterious variants. Erroneous classification of a genetic variant may have a great effect on at-risk familial who undergo genetic testing for risk prediction because it results in incorrect clinical recommendations.

LS is the most common hereditary CRC-predisposing syndrome and accounts for $3 \%$ of unselected CRC cases. A significant proportion of DNA variations found in patients suspected of having LS are UVs $(32 \%, 18 \%$ and $38 \%$ for $M L H 1, M S H 2$ and $M S H 6$, respectively) [6]. The pathogenicity of the MLH1 p.Lys618Ala variant remains controversial because of conflicting data [InSiGHT, http://www.insight-group.org] (Figure 5).

The p.Lys618Ala substitution replaces a charged amino acid with a neutral one, and occurs alongside four charged amino acids that are well conserved in mammals. In silico predictions of the pathogenicity of this variant using the PolyPhen http://genetics.bwh.harvard.edu/pph/ and SIFT http://sift.jcvi.org/ computational program

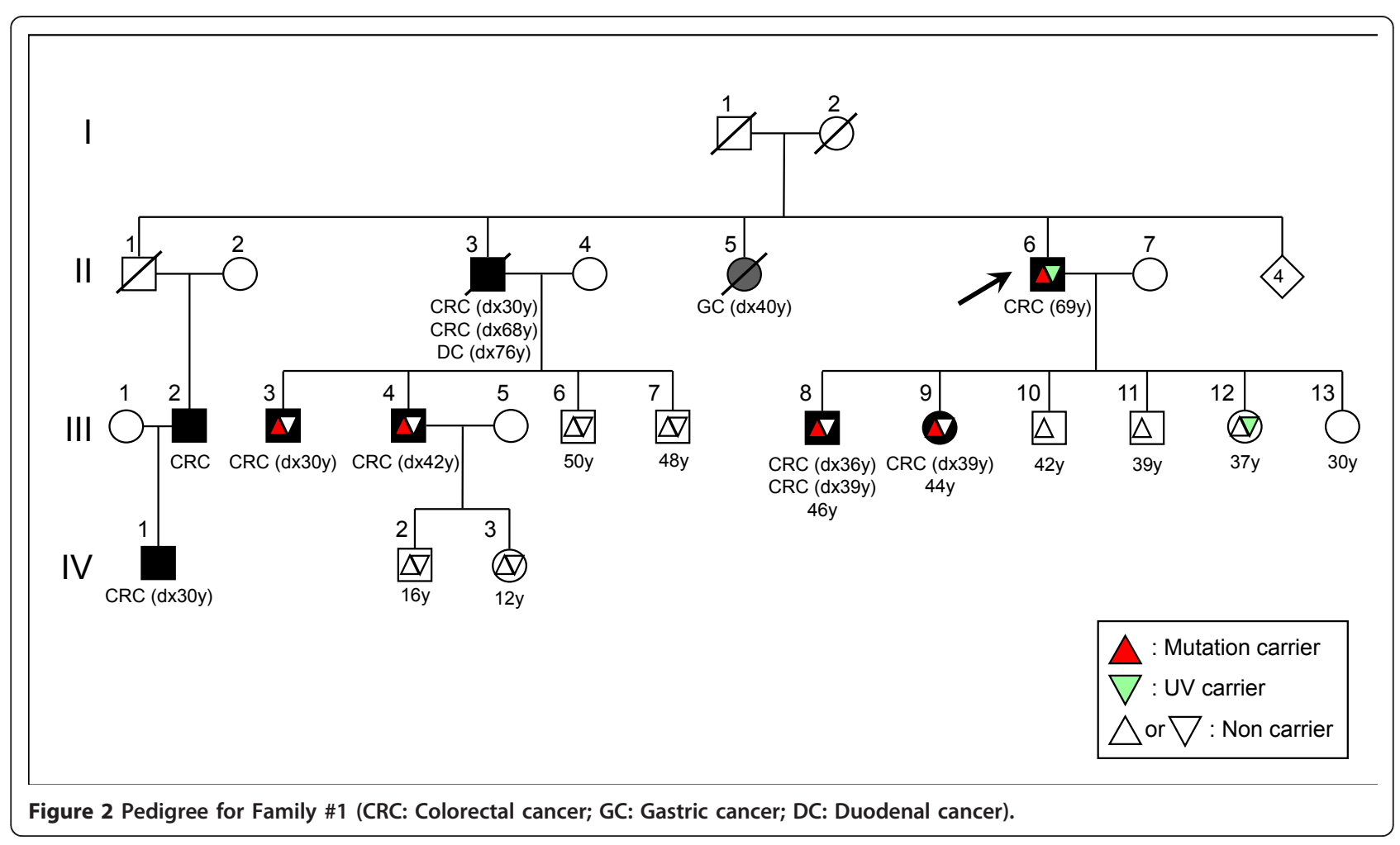




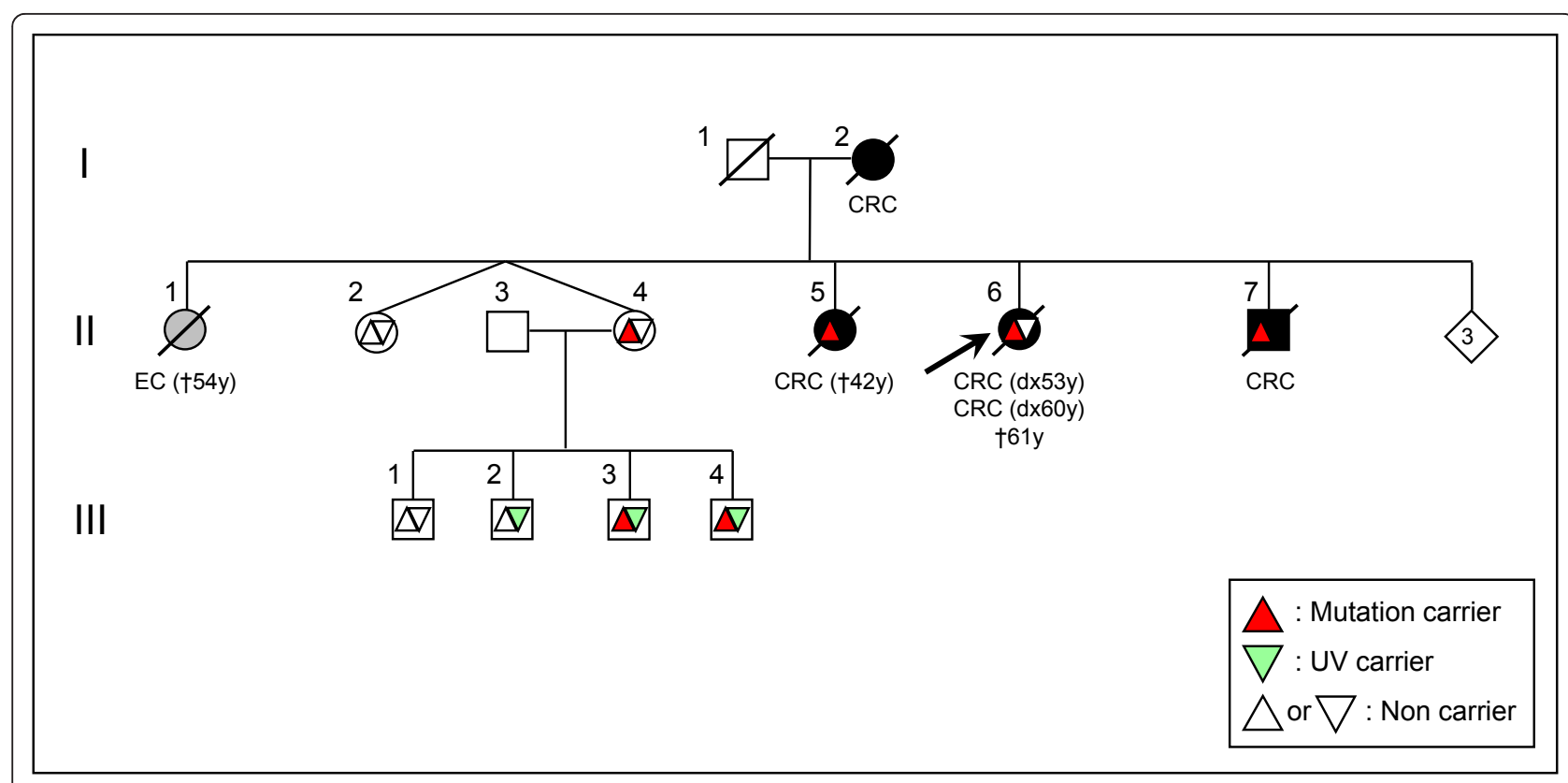

Figure 3 Pedigree for Family \#2 (CRC: Colorectal cancer; EC: Endometrial cancer).

were discordant; the SIFT analysis classified it as a tolerant variant and the PolyPhen analysis classed it as possibly damaging [9]. It has been shown that this variant may reduce the binding ability of MLH1 to PMS2 in HCT116 cells co-transfected with mutated $M L H 1$ and wild-type PMS2 [10]. In contrast, it had no effect on the ability of MLH1 to bind PMS2 in a co-immunoprecipitation assay
[9]. Functional analysis using the pCAS ex vivo splicing assay and RNA analysis also demonstrated no effect [11]. Moreover, a significant decrease in MLH1 protein stability has been found for the p.Lys618Ala variant [9].

The results of in silico prediction and functional assays alone are insufficient to determine whether this variant is deleterious or a rare functional polymorphism.

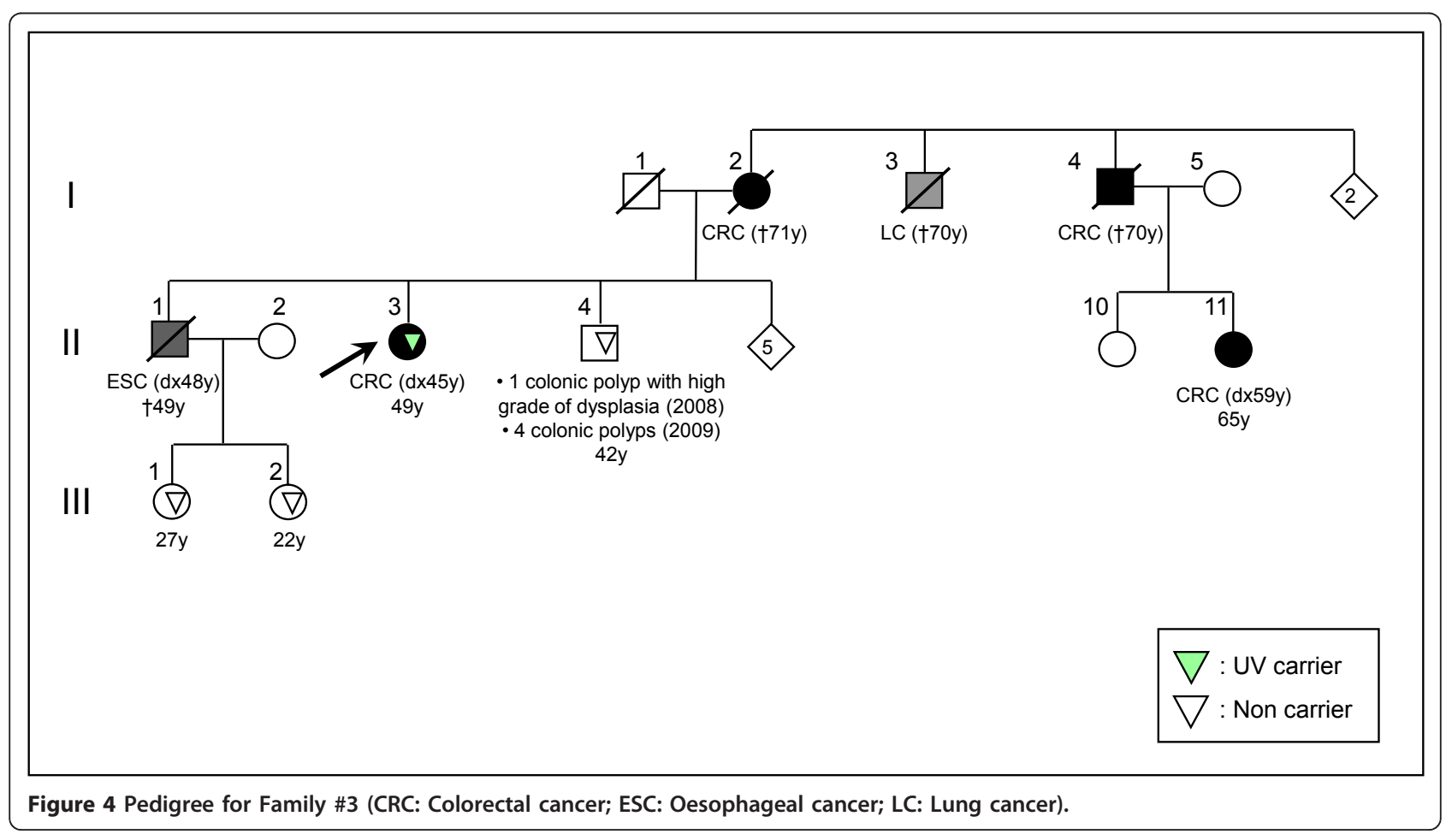




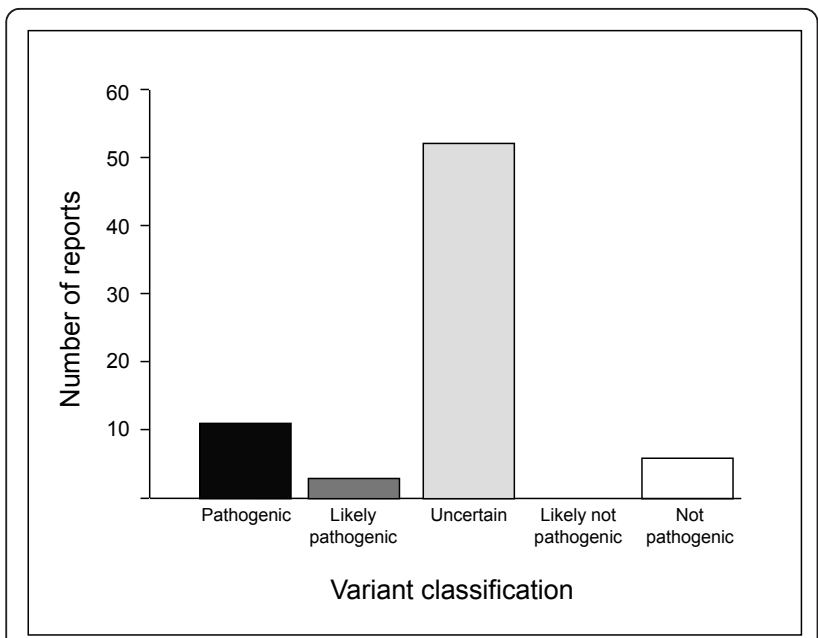

Figure 5 Classification of the MLH1 p.Lys618Ala variant according to the InSiGHT database (accessed on 07/2010).

For this purpose, it is necessary to integrate indirect evidence with direct genetic evidence involving clinical observations of disease occurrence.

The frequency of variants in unaffected controls is used often to distinguish between neutral and potentially deleterious variants. If the frequency of a variant among a few hundred controls is $\geq 1 \%$, it is highly unlikely to be a high-risk variant. In such cases, it is still possible that the variant will be associated with a modest risk of the disease [3]. Case-control studies enable quantification of the disease risk associated with the variant. The main disadvantage of such analyses is that a large sample size is required to obtain sufficient power to detect the lower risk level. The sample size required is related inversely to the frequency of the variant in the population. The sample size used in the present study resulted in $80 \%$ power to detect an OR of 3.0 (two-sided test; alpha level, $5 \%$ ). The frequency of the p.Lys618Ala variant in our control series was $2.7 \%$ and no significant differences were observed in the sporadic and familial groups, indicating that a high penetrance effect for colorectal carcinogenesis can be excluded. Similar results were reported in case-control studies on Scottish and Danish populations $[12,13]$.

As most disease pedigrees are small, it is difficult to obtain a sufficient number of samples from affected and informative unaffected individuals. Moreover, LS deleterious variants are not completely penetrant. For these reasons, it is rarely possible to categorize variants as deleterious based on segregation alone. The co-occurrence of another known deleterious variant reduces the likelihood that an UV is truly deleterious, especially when both variants are located in trans [3]. To our knowledge, co-occurrence of a deleterious variant in one of the LS genes with the p.Lys618Ala variant has been observed in only two families. Liu et al [14] described an index subject from a LS family with two heterozygous variants (c.546-2A $>\mathrm{G}$ and c.1852_1853AA $>\mathrm{GC}$ ); only the former segregated with LS in the family. Similarly, Steinke et al [15], described the co-occurrence of the p.Lys618Ala (c.1852_1853AA $>\mathrm{GC}$ ) variant with the MSH6 p.Arg1068X (c.3202C $>$ T) deleterious variant.

Herein, we describe the coexistence of the p.Lys618Ala variant with deleterious variants in another two unrelated LS families. In one family, the allele distribution of the pathogenic and unclassified variant was in trans, in the other family the pathogenic variant was detected in the MSH6 gene and only the deleterious variant co-segregated with the disease in both families. This evidence indicates that the p.Lys618Ala variant is not deleterious.

The molecular hallmark of LS tumours is an MSI phenotype, a functional consequence of MMR deficiency. It is expected that the putative germ-line mutation responsible for LS would confer the MSI phenotype. We tested the MSI status of 17 tumours from p.Lys618Ala carriers and detected only two cases of MSI (11.8\%). Taking into consideration the bias caused by the over-representation of Bethesda Criteria-positive tumours in this subset of cases (8/17), the MSI frequency was not significantly different from that in the unselected CRC group [7]. This is further proof that the presence of this variant is irrelevant to the functional inactivation of $M L H 1$ in CRC patients.

Nonetheless, we cannot exclude the possibility that this variant may result in a small increase in susceptibility to CRC or adenomas, as was suggested by Fearnhead et al [16]. Further studies with appropriate sample sizes are required to address the low penetrance effect of this variant in CRC.

Finally, we hypothesize that the clinical significance of a genetic variant may differ according to genetic background. Gene functionality may be the net result of the effects of allelic structures and their interactions with environmental factors. It is possible that low-penetrance variants behave differently in different populations, making it difficult to make predictions in terms of conferred risk.

\section{Conclusions}

Taken together, the results of this study and others indicate that the c.1852_1853AA>GC variant should be considered a neutral variant for LS. These findings have considerable relevance for the clinical management of CRC probands and their relatives.

\section{Acknowledgements}

We are indebted to the patients and their families. We thank all members of the Hereditary Cancer Program of the Comunidad Valenciana, Spain and the EPICOLON-II Consortium. This action has been supported in part by grants from the Generalitat Valenciana in Spain (AP140/08) and the Biomedical Research Foundation from the Hospital of Elche, Spain (FIBElx0902). CG, AMC, CEl and LPC are recipients of fellowships from the Conselleria de 
Educació (Generalitat Valenciana); Fundacion Juan Peran-Pikolinos; Fundacion Carolina-BBVA and Fondo Investigación Sanitaria (Fl07/00303), respectively. RJ is receptor of a grant from Instituto de Salud Carlos III (INT09/208).

\section{Author details}

${ }^{1}$ Laboratorio Investigación. Hospital Universitario Elche. Elche. Spain. ${ }^{2}$ Laboratorio Investigación. Hospital Universitario Alicante. Alicante. Spain. ${ }^{3}$ Unidad Consejo Genético Cáncer. Hospital Universitario Elche. Elche. Spain. ${ }^{4}$ Unidad Consejo Genético Cáncer. Hospital Universitario La Fe. Valencia. Spain. ${ }^{5}$ Unidad Biopatología Molecular. Hospital Provincial Castellón. Castellón. Spain. ${ }^{6}$ Servicio Anatomía Patológica. Hospital La Plana Castellón. Castellón. Spain. 'Fundación Pública Galega de Medicina Xenómica, CIBERER, Grupo de Medicina Xenómica-Universidad de Santiago de Compostela. Santiago de Compostela. Spain. ${ }^{8}$ Hospital Donostia. Instituto Biodonostia. Universidad del País Vasco. CIBERehd. San Sebastián. Spain. ${ }^{9}$ Departamento de Gastroenterología. Hospital del Mar. Barcelona. Spain. ${ }^{10}$ Departmento de Gastroenterología. Institut de Malaties Digestives, Hospital Clínic, IDIBAPS, CIBERehd, Universidad de Barcelona. Barcelona. Spain. ${ }^{11}$ Department of Medicine and Cancer Center. University of Illinois at Chicago. Chicago, II. USA. ${ }^{12}$ Servicio de Medicina Digestiva. Hospital Universitario La Fe. Valencia. Spain. ${ }^{13}$ Servicio de Anatomía Patológica. Hospital Universitario Alicante. Alicante. Spain. ${ }^{14}$ Unidad de Gastroenterología. Hospital Universitario Alicante. Alicante. Spain.

\section{Authors' contributions}

ACastillejo participated in the design and coordination of the study, in the molecular genetic studies and helped to draft the manuscript. CG, AMC, $V M B, C E, M I C, L P C, C R P$ and ACarracedo participated in the molecular genetic studies. ABSH, AS, LB, MA and ACastells participated in the design of the study, performed the statistical analysis and helped to draft the manuscript. EO, RL, XL, JC and RJ participated in the biobanking of samples and helped to draft the manuscript. CA and AP participated in the pathological analysis of the samples and helped to draft the manuscript. JLS conceived the study, participated in its design and coordination and drafted the manuscript. All authors read and approved the final manuscript.

\section{Competing interests}

The authors declare that they have no competing interests.

Received: 13 September 2010 Accepted: 19 January 2011

Published: 19 January 2011

\section{References}

1. Plon SE, Eccles DM, Easton D, Foulkes WD, Genuardi M, Greenblatt MS, Hogervorst FB, Hoogerbrugge N, Spurdle AB, Tavtigian SV, IARC Unclassified Genetic Variants Working Group: Sequence variant classification and reporting: recommendations for improving the interpretation of cancer susceptibility genetic test results. Hum Mutat 2008, 29:1282-1291.

2. Kryukov GV, Pennacchio LA, Sunyaev SR: Most rare missense alleles are deleterious in humans: implications for complex disease and association studies. Am J Hum Genet 2007, 80:727-739.

3. Goldgar DE, Easton DF, Byrnes GB, Spurdle AB, Iversen ES, Greenblatt MS, IARC Unclassified Genetic Variants Working Group: Genetic evidence and integration of various data sources for classifying uncertain variants into a single model. Human Mutat 2008, 29:1265-1272.

4. Lynch HT, de la Chapelle A: Hereditary colorectal cancer. N Engl J Med 2003, 348:919-932

5. Lynch HT, Boland CR, Gong G, Shaw TG, Lynch PM, Fodde R, Lynch JF, de la Chapelle A: Phenotypic and genotypic heterogeneity in the Lynch syndrome: diagnostic, surveillance and management implications. Eur $J$ Hum Genet 2006, 14:390-402.

6. Peltomaki P, Vasen H: Mutations associated with HNPCC predispositionUpdate of ICG-HNPCC/INSiGHT mutation database. Dis Markers 2004, 20:269-276.

7. Piñol V, Castells A, Andreu M, Castellví-Bel S, Alenda C, Llor X, Xicola RM, Rodríguez-Moranta F, Payá A, Jover R, Bessa X, Gastrointestinal Oncology Group of the Spanish Gastroenterological Association: Accuracy of revised Bethesda guidelines, microsatellite instability, and immunohistochemistry for the identification of patients with hereditary nonpolyposis colorectal cancer. JAMA 2005, 293:1986-1994.
8. Buhard O, Cattaneo F, Wong YF, Yim SF, Friedman E, Flejou JF, Duval A, Hamelin R: Multipopulation analysis of polymorphisms in five mononucleotide repeats used to determine the microsatellite instability status of human tumors. J Clin Oncol 2006, 24:241-251.

9. Perera S, Bapat B: The MLH1 variants p.Arg265Cys and p.Lys618Ala affect protein stability while p.Leu749GIn affects heterodimer formation. Hum Mutat 2008, 29:332.

10. Belvederesi L, Bianchi F, Loretelli C, Gagliardini D, Galizia E, Bracci R, Rosati S, Bearzi I, Viel A, Cellerino R, Porfiri E: Assessing the pathogenicity of $\mathrm{MLH} 1$ missense mutations in patients with suspected hereditary nonpolyposis colorectal cancer: correlation with clinical, genetic and functional features. Eur J Hum Genet 2006, 14:853-859.

11. Tournier I, Vezain M, Martins A, Charbonnier F, Baert-Desurmont S, Olschwang S, Wang Q, Buisine MP, Soret J, Tazi J, Frébourg T, Tosi M: A large fraction of unclassified variants of the mismatch repair genes MLH1 and MSH2 is associated with splicing defects. Hum Mutat 2008, 29:1412-1424.

12. Barnetson RA, Cartwright N, van Vliet A, Haq N, Drew K, Farrington S, Williams N, Warner J, Campbell H, Porteous ME, Dunlop MG: Classification of ambiguous mutations in DNA mismatch repair genes identified in a population-based study of colorectal cancer. Hum Mutat 2008, 29:367-374.

13. Christensen LL, Madsen BE, Wikman FP, Wiuf C, Koed K, Tjønneland A, Olsen A, Syvänen AC, Andersen CL, Orntoft TF: The association between genetic variants in $\mathrm{hMLH} 1$ and $\mathrm{hMSH} 2$ and the development of sporadic colorectal cancer in the Danish population. BMC Med Genet 2008, 9:52.

14. Liu T, Tannergard P, Hackman P, Rubio C, Kressner U, Lindmark G, Hellgren D, Lambert B, Lindblom A: Missense mutations in hMLH1 associated with colorectal cancer. Hum Genet 1999, 105:437-441.

15. Steinke V, Rahner N, Morak M, Keller G, Schackert HK, Görgens H, Schmiegel W, Royer-Pokora B, Dietmaier W, Kloor M, Engel C, Propping P, Aretz S, German HNPCC Consortium: No association between MUTYH and MSH6 germline mutations in 64 HNPCC patients. Eur J Hum Genet 2008, 16:587-592.

16. Fearnhead NS, Wilding JL, Winney B, Tonks S, Bartlett S, Bicknell DC Tomlinson IP, Mortensen NJ, Bodmer WF: Multiple rare variants in different genes account for multifactorial inherited susceptibility to colorectal adenomas. Proc Natl Acad Sci USA 2004, 101:15992-15997.

\section{Pre-publication history}

The pre-publication history for this paper can be accessed here: http://www.biomedcentral.com/1471-2350/12/12/prepub

\section{doi:10.1186/1471-2350-12-12}

Cite this article as: Castillejo et al: Evidence for classification of c.1852_1853AA>GC in MLH1 as a neutral variant for Lynch syndrome. BMC Medical Genetics 2011 12:12.

\section{Submit your next manuscript to BioMed Central and take full advantage of:}

- Convenient online submission

- Thorough peer review

- No space constraints or color figure charges

- Immediate publication on acceptance

- Inclusion in PubMed, CAS, Scopus and Google Scholar

- Research which is freely available for redistribution 